\title{
Hard Tissue Analysis of NMR after Fluoride Administration
}

\author{
Hye-Young Kim ${ }^{1}$, Seoul-Hee $\mathrm{Nam}^{1 *}$, and Man-Seok Han ${ }^{2 *}$ \\ ${ }^{1}$ Dept. of Dental Hygiene, Kangwon National University, Samcheok, Republic of Korea \\ ${ }^{2}$ Dept. of Radiological Science, Kangwon National University, Samcheok, Republic of Korea
}

(Received 6 October 2016, Received in final form 7 November 2016, Accepted 15 November 2016)

\begin{abstract}
Fluoride (F) is an important element for the mineralization of body tissues. The purpose of this study was to administer fluoride prenatally to rats to evaluate its beneficial concentration for rat bone using microstructural analysis, to analyze its effect on the bone structure, and to evaluate the effect of its transfer through rat placenta. Fourier transform infrared spectroscopy (FTIR) and nuclear magnetic resonance spectrometry (NMR) were performed. The ${ }^{19} F$ NMR and ${ }^{31} \mathrm{P}$ NMR signals suggested the existence of fluoride ions in the apatite lattice because the signals were caused by the fluoride ions that were coupled to the phosphate atoms and were affected in the phosphate phases other than the element phases in the apatite. Consequently, if it was not affected too much, the desirable concentration of prenatal fluoride treatment could have a helpful effect on the bone crystal structure through placental fluoride transfer.
\end{abstract}

Keywords: Fourier transform infrared spectroscopy (FTIR), nuclear magnetic resonance spectrometry (NMR), microstructural analysis

\section{Introduction}

The fluoridation of community drinking water is considered one of the ten most important public health achievements of the 20th century [1] and the most costeffective of all the community-based dental caries prevention methods [2]. Despite its documented effectiveness in preventing dental caries $[3,4]$, however, the effects of continuous systemic fluoride $(\mathbf{F})$ use are not completely understood. Specifically, the effects of different fluoride concentrations on enamel and dentin mineral are unknown.

According to the World Health Organization (WHO) standards, the permissible level of fluoride in drinking water is $1 \mathrm{ppm}$ [5]. The wide use of fluoride in drinking water (at an approximately $1 \mathrm{ppm}$ concentration) and in dental products is beneficial for reducing dental caries [6]. The protective effect of fluoride against dental caries is usually attributed to its capacity to influence enamel formation in such a way that the enamel surface becomes more resistant to acid dissolution. Caldera et al. [7]

CThe Korean Magnetics Society. All rights reserved.

*These authors contributed equally to this work.

Seoul-Hee Nam. Tel: +82-33-540-3394, Fax: +82-33-540-3399

e-mail:miss4228@naver.com

Man-Seok Han. Tel: +82-33-540-3383, Fax: +82-33-540-3389

e-mail: angio7896@naver.com reported the presence of fluoride in 70 and $40 \%$ of deciduous teeth, respectively, after pre- and postnatal use only of fluoride supplements.

Babeaux and Zipkin [8] did not observe differences in the calcium and phosphorus contents of the bone ash of human beings who had drunk water containing up to 4 ppm fluoride. Eanes and Meyer [9] have shown the same in vitro in the case of octacalcium phosphate (OmaCP), which can occur as a transitional crystalline phase in invitro apatite formation. Grynpas and Rey [10] showed that fluoride influences the bone crystal structure, and it is feasible to postulate that fluoride may also influence the tooth apatite crystals. Vieira et al. [11] showed a correlation between the tooth fluoride concentration and the crystallite size. Baud et al. [12] showed that in the bone, substantial increases in the width of apatite crystal and lattice perfection accompany fluoride uptake. Sakong et al. [13] suggested that $1 \mathrm{ppm}$ fluoride could increase the surface microhardness and calcium content of the bone better than 10 and $100 \mathrm{ppm}$ fluoride could. No information relating to the tooth fluoride concentration and the tooth (enamel and dentin) crystallite size, however, is currently available.

One of the most important advantages of solid-state nuclear magnetic resonance spectrometry (NMR) is the ability to directly observe any element in its chemical 
environment in the sample under study, irrespective of whether the sample is crystalline or not [14]. In the bone, it is possible to observe the environment of a phosphorous or carbon atom and to determine whether the atom occupies a position associated with a hydroxyapatite lattice of another lattice type or it is a disorder around a lattice position [15]. NMR is thus useful in providing structural information that may not be obtainable from Xray diffraction $(\mathrm{XRD})$ either because the material does not have definite resolved diffraction peaks or because phases are present in quantities too small to be detected by Xrays. NMR also provides information about the presence and mobility of hydrogen atoms that are not detectable by XRD. Lee et al. [16] suggested that NMR could be a helpful adjunct to XRD in ascertaining the state of digenesis of archaeological bone. Studies of ${ }^{19} \mathrm{~F}$ NMR in fluoridated bone mineral have been carried out. Previously, Ebifegha et al. [17] observed weak and rapidly decaying FID and echo signals in fluoridated rat bone powder. Gelman and Code [18] argued that the ${ }^{19} \mathrm{~F}$ spins in bone mineral typically experience weaker heteronuclear dipolar coupling than those in the mineral hydroxyapatite, which is often considered a prototype of bone mineral. Pan [19] reported that at fluoride-treated hydroxyapatite, the bone showed the formation of HA with a surface coverage of less than one unit cell layer.

Thus, the purpose of this study was to prenatally administer fluoride to rats to evaluate its beneficial concentration for the bone using microstructural analysis, and to analyze the effect of fluoride on the bone structure.

\section{Materials and Methods}

\subsection{Animals and Grouping}

Fifteen 8-week-old female rats (initial average weight: 180-200 g) were randomly divided into five groups (one control group and four experimental groups), with three rats in each group. They were mated with 10-week-old male rats (1:1). During the mating period, redistilled water was administered to the control group while redistilled water containing 1/10/20/100 ppm fluoride was administered to the experimental groups. After delivery, the litters were housed along with their mothers for 3 weeks, and were then housed for another week following separation. At 4 weeks after delivery, all the young rats were sacrificed, and their femurs were extracted.

\subsection{Microstructural analysis: NMR}

${ }^{31} \mathrm{P}$ and ${ }^{19} \mathrm{~F}$ NMR analyses were carried out using a spectrometer (400 MHz Solid-State FT-NMR Spectrometer, DSX $400 \mathrm{MHz}$, Bruker Analytische GmbH, Germany) equipped with a $5 \mathrm{~mm}$ fast-spinning probe. The magicangle spinning rate was $12 \mathrm{kHz}$. The $\mathrm{F}$ and $\mathrm{P}$ chemical shifts were referenced to an external solution of $\mathrm{C}_{6} \mathrm{~F}_{6}$ and $85 \% \mathrm{H}_{3} \mathrm{PO}_{4}(0 \mathrm{ppm})$, respectively. The ${ }^{31} \mathrm{P}$ NMR spectrum of synthetic $\mathrm{Ca}_{5}\left(\mathrm{PO}_{4}\right)_{3} \mathrm{~F}_{0.25} \mathrm{OH}_{0.75}$ was noted.

The spectral resonance frequency (V0) was calculated via NMR using the following formula:

$$
\begin{aligned}
V 0 & =\frac{V}{2 \pi} B 0 \\
r \quad & =\text { gyromagnetic ratio } \\
B 0 & =\text { magnetic field strength }
\end{aligned}
$$

The chemical shift (ppm) was also calculated using the following formula:

$$
\text { Chemical shift }(\mathrm{ppm})=\frac{V i-V^{\text {ref }}}{V 0} \times 1,000,000
$$

$V 0=$ resonance frequency of the chemical bonds that were not nuclear

$V i=$ resonance frequency of each element in the molecule $V^{\text {ref }}=$ reference frequency

\section{Results}

\subsection{Microstructural analysis}

The ${ }^{19} \mathrm{~F}$ NMR spectrum showed around $80-90 \mathrm{ppm}$ resonance (Fig. 1). These peaks were assigned to the
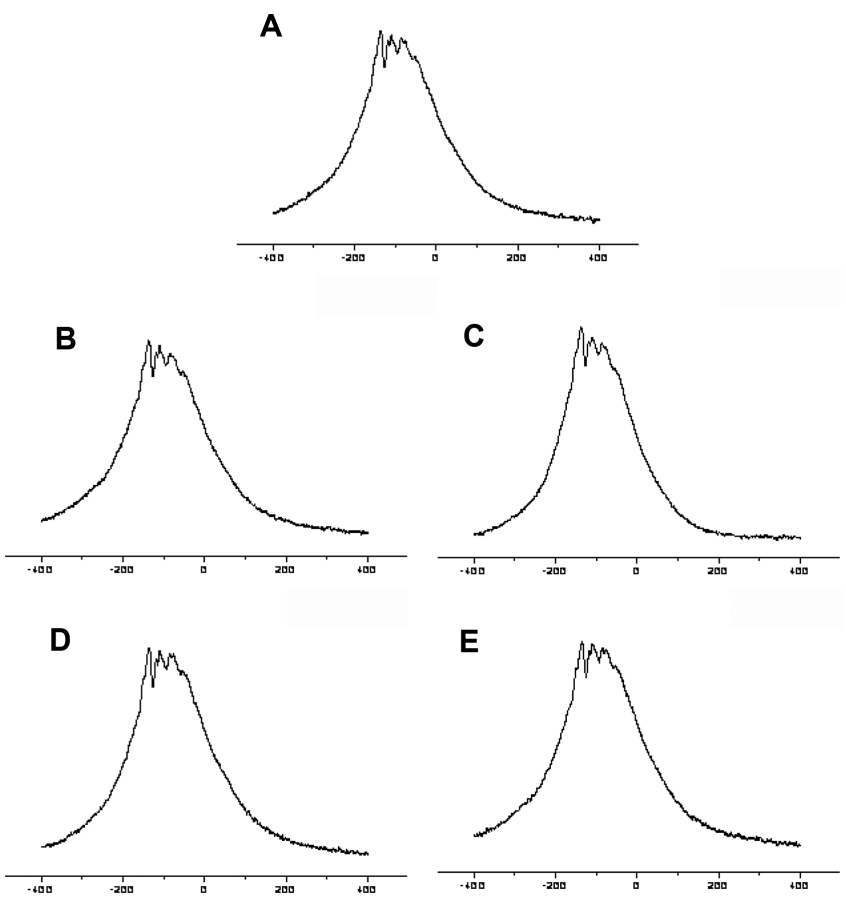

Fig. 1. Bone crystal structure by placental fluoride transfer. ${ }^{19} \mathrm{~F}$ NMR spectra: (A) control; (B) 1 ppm; (C) 10 ppm; (D) 20 ppm; and (E) 100 ppm 


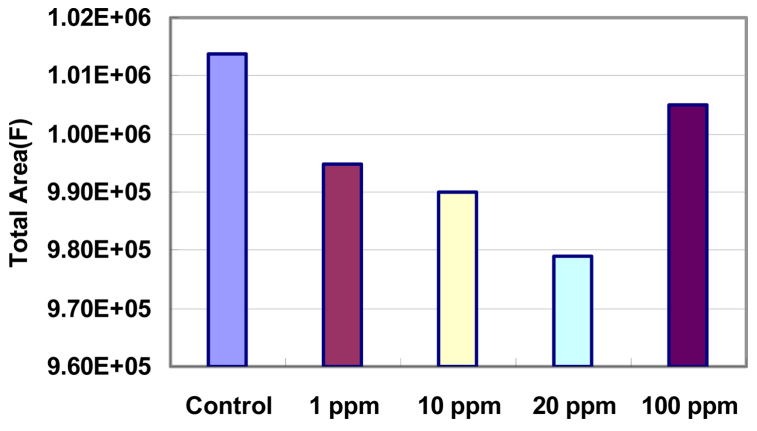

Fig. 2. (Color online) Total peak area of the bone ${ }^{19} \mathrm{~F}$ NMR spectra according to the concentration of the administered fluoride.

hydroxyapatite with low fluoride substitutions, and indicated the presence of fluorine in the apatite. All the peaks were broad and could not be compared using the naked eye according to the concentration of the administered fluoride.

The total peak areas decreased with increasing concentrations of administered prenatal fluoride (Fig. 2). The spectra became sharper when the concentrations increased, and became broader with decreasing intensity peaks at $100 \mathrm{ppm}$.

The ${ }^{31} \mathrm{P}$ NMR spectra showed around 4 and $7 \mathrm{ppm}$ resonances (Fig. 3). These peaks indicated the presence of at least two different phosphate species in the apatite. The small shoulder peaks (around $4 \mathrm{ppm}$ ) collapsed with the increasing concentrations of administered prenatal fluoride,
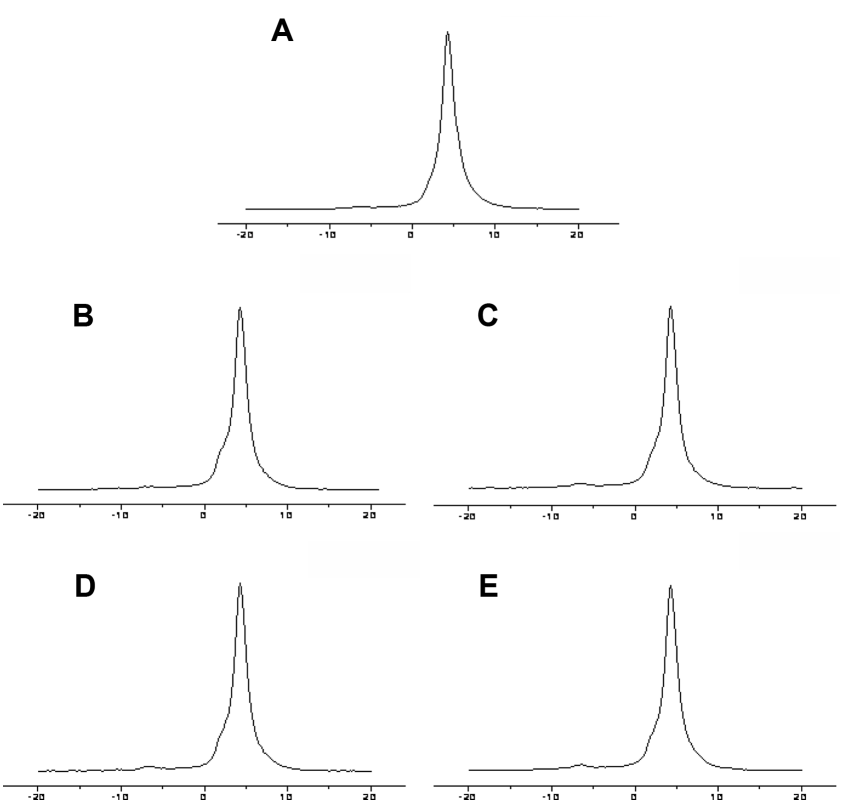

Fig. 3. Bone crystal structure by placental fluoride transfer. ${ }^{31}$ P NMR spectra (A) control; (B) 1 ppm; (C) 10 ppm; (D) 20 ppm; and (E) 100 ppm

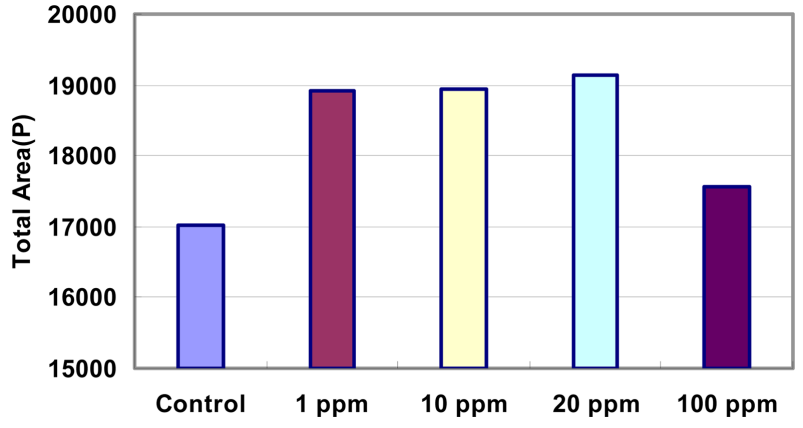

Fig. 4. (Color online) Total area of the bone ${ }^{31} \mathrm{P}$ NMR spectra according to the concentration of the administered fluoride.

and the 1,10 , and $20 \mathrm{ppm}$ groups had similar tendencies. The total peak areas also increased with increasing concentrations of administered prenatal fluoride (Fig. 4).

The spectra sharpened with increased peak intensities when the concentration of the administrated prenatal fluoride increased, and became broader with decreased intensity peaks at $100 \mathrm{ppm}$.

\section{Discussion}

Fluoride is important for bone health and plays an important role in normal bone mineralization. The current research is focused on the possible role of prenatal fluoride in relation to the placental barriers. Although it has been conclusively demonstrated in different species that fluoride crosses the placental barrier and is taken up by the fetal tissues, the literature concerning fluoride transfer and feto-maternal metabolism remains contradictory [20-22]. The fluoride-induced increase in the crystallinity of bone apatite may be accompanied by a drop in the effective surface area per unit of mass. Eanes and Hailer [23] suggested that the fluoride ions stimulate seed crystal growth in an anisotropic manner, similar to that observed in the adult human bone apatite. This finding suggests that the latter growth in vivo was partly the consequence of the direct fluoride ion-mineral interactions.

As noted above, NMR data are useful for obtaining structural information that may be unavailable from XRD data either because the material does not give sharp resolved diffraction peaks or because the quantities of the phases are too small to be detected via XRD. Furthermore, NMR data provide information about the presence and mobility of hydrogen atoms, which cannot be detected by XRD. Therefore, the magic-angle spinning NMR techniques, which have been very useful for many systems, were used in this study. Pan [24] reported that the application on fluoride-treated hydroxyapatite, the principal inorganic constituent of the dental enamel and dentine, showed the 
formation of fluorohydroxyapatite with less than one unit cell layer surface coverage.

In the experiments that were conducted in this study, ${ }^{19} \mathrm{~F}$ NMR peaks were assigned to hydroxyapatite (HA) with low fluoride substitution, which indicated the presence of fluorine in the apatite. Generally, the peak at around $58-59 \mathrm{ppm}$ is assigned to fluorohydroxyapatite [25]. In this study, however, the ${ }^{19} \mathrm{~F}$ NMR peaks were observed at around $80-90 \mathrm{ppm}$ resonance. The spectra sharpened with the increase in the concentration of administered prenatal fluoride, whereas for the $100 \mathrm{ppm}$ fluoride group, the spectra broadened and the intensity of the peak decreased.

The observed ${ }^{19} \mathrm{~F}$ NMR signals suggested the existence of a fluoride ion in the apatite lattices because the NMR signal was caused by the fluoride ions coupled to the phosphate atoms and thus affected only the phosphate phase and not the phases of the other elements in the apatite.

The present findings demonstrated that fluoride crosses the placental barrier and is absorbed by the fetus. It is important to note that because the placenta is a physiological membrane, the amount of fluoride reaching the fetus would be dependent on the dose ingested during pregnancy. In other words, the results of this study suggest that all the changes induced by the direct incorporation of the fluoride ions into the apatite lattice, especially in the hydroxyapatite structures, carbonate, phosphate, and amide unit cell volume, were affected by the prenatal fluoride administration.

The aforementioned findings in the animal model presented herein, however, have limited human application. It is clear from the study results that fluoride is incorporated into the bone mineral with the administration of prenatal fluoride to the mother. Prospective and long-term studies are required to evaluate the changes in the bone mineral components caused by varying fluoride concentrations.

\section{Conclusion}

The ${ }^{19} \mathrm{~F}$ NMR and ${ }^{31} \mathrm{P}$ NMR signals suggested the existence of fluoride ions in the apatite lattice because the NMR signal was caused by the fluoride ions coupled to the phosphate atoms and thus affected only the phosphate phase and not the phases of the other elements in the apatite. These results indicate that such incorporation of fluoride ions is related to the strengthening of the bone crystal structure. Consequently, the appropriate prenatal administration of fluoride can have a beneficial effect on the bone crystal structure owing to the placental fluoride transfer.

\section{References}

[1] E. T. Everett, M. A. McHenry, N. Reynolds, H. Eggertsson, J. Sullivan, C. Kantmann, E. A. Martinez-Mier, J. M. Warrick, and G. K. Stookey, J. Dent. Res. 81, 794 (2002).

[2] T. M. Marthaler, Oral. Health Prev. Dent. 1, 129 (2003).

[3] J. J. Murray, Caries Res. 27, 2 (1993).

[4] L. W. Ripa, J. Public Health Dent. 53, 17 (1993).

[5] WHO. The health aspects of food and nutrition. A manual for developing countries in western pacific region, 3rd edition, Western Pacific Regional Office (1979) pp. 27-60.

[6] S. Jones, B. A. Burt, P. E. Petersen, and M. A. Lennon, Bull. World Health Organ. 83, 670 (2005).

[7] R. Caldera, J. Chavinie, J. Fermanian, D. Tortrat, and A. M. Laurent, Biol. Neonate 54, 263 (1988).

[8] W. L. Babeaux and I. Zipkin, J. Oral. Ther. Pharmacol. 3, 124 (1966).

[9] E. D. Eanes and J. L. Meyer, J. Dent. Res. 57, 617 (1978).

[10] M. D. Grynpas, C. Rey, Bone. 13, 423 (1992).

[11] A. Vieira, R. Hancock, H. Limeback, M. Schwartz, and M. Grynpas, J. Dent. Res. 82, 909 (2003).

[12] C. A. Baud, J. M. Very, and B. Courvoisier, Bone. 9, 361 (1988).

[13] Y. Sakong, J. Y. Kim, J. S. Song, Y. H. Kwon, and K. B. Song, J. Korean Acad. Dent. Health 27, 139 (2003).

[14] J. Timanen, L. Alvila, P. Hirva, and T. T. Pakkanen, J. Mat. Sci. 9, 187 (1998).

[15] M. I. Kay, R. A. Young, and A. S. Posner, Nature 204, 1050 (1964).

[16] A. P. Lee, J. Klinowski, and E. A. Marseglia, J. Archae Sci. 22, 257 (1995).

[17] M. E. Ebifegha, J. E. Harrison, K. G. McNeill, S. S. Krishnan, and J. Ssengabi, Int. J. Rad. Appl. Instrum. 37, 159 (1986).

[18] N. Gelman and R. F. Code, Solid State Nucl. Magn. Reson. 143-4, 191 (1999).

[19] Y. Pan, Solid State Nuclear Magn. Res. 5, 263 (1995).

[20] W. S. Driscoll, J. Dent. Child 48, 109 (1981).

[21] T. F. Collins, R. L. Sprando, T. N. Black, M. E. Shackelford, N. Olejnik, M. J. Ames, J. I. Rorie, and D. I. Ruggles, Food Chem. Toxicol. 39, 867 (2001).

[22] A. S. Al-Hiyasat, A. M. Elbetiecho, and H. Darmani, Fluoride 33, 9 (2000).

[23] E. D. Eanes and A. W. Hailer, Calcif. Tissue Int. 63, 250 (1998).

[24] Y. Pan, Solid State Nuclear Magnetic Resonance 5, 263 (1995).

[25] M. Iijima, D. G. Nelson, Y. Pan, A. T. Kreinbrink, M. Adachi, T. Goto, and Y. Moriwaki, Calcif. Tissue Int. 59, 377 (1996). 